\title{
CONCEPCIONES DE LOS PROFESORES DE EDUCACIÓN SECUNDARIA SOBRE EVALUACIÓN
}

Leonor Buendía Eisman'

Marcelo Carmona Fernández

Daniel González González

Rafael López Fuentes

Facultad de Ciencias de la Educación

Universidad de Granada

\section{LAS CONCEPCIONES DE LOS PROFESORES: FUNDAMENTOS TEÓRICOS}

Hoy, nos parece fuera de duda considerar el importante peso que lo social y cultural tiene, tanto en ver el mundo como lo vemos como en la construcción de los instrumentos que utilizamos para pensarlo como lo pensamos. Cuando tratamos de acercarnos a la conceptualización de las concepciones, este fuerte componente aparece necesariamente.

No debemos olvidar que "fueron las circunstancias de la vida social del hombre primitivo, el pertenecer a una comunidad humana con interacciones complejas y su necesidad de ayudarse mientras al mismo tiempo ayuda a los demás, las que, más que nada, hicieron al hombre como especie, la criatura penetrante y astuta que hoy conocemos" (Humphrey, 1987: 14). La perspectiva de Humphrey, diferente a las posiciones tradicionales, se basa en afirmar

\footnotetext{
' Este trabajo forma parte de la investigación subvencionada por el MEC: CIDE, en el Concurso Nacional para otorgar Ayudas a la Investigación Educativa (Resolución 6 de marzo de 1996/BOE de 18 de marzo).
} 
que lo que caracteriza a nuestra especie no es tanto la capacidad para inventar tecnología, como de transmitirla de forma acumulativa.

Esta astucia psicológica, para el ámbito de lo social, ha sido destacada también por Cosmides (1989) y Riviere (1991). En el frontispicio de lo que se transmite de forma acumulativa se encuentran las concepciones sobre nosotros mismos, las formas en cómo entendemos que se accede a ser, cómo nos concebimos y los problemas que encontramos en cualquier ámbito en el que nos fijemos.

Desde una perpectiva amplia, tres conjuntos de elementos componen la piedra angular sobre la que se asientan las concepciones humanas que nosotros estudiaremos aquí. En primer lugar, hay un eje de naturaleza bio-antropológica que hace referencia a las consecuencias que se derivan de nuestra condición como seres vivos, surgidos de un largo proceso de evolución y su influencia en la forma de conocer. En segundo lugar, existe una amplia experiencia como resultado de nuestra naturaleza histórica. Por último, encontramos un conjunto de elementos derivados de nuestra particularidad psicológica. En cada uno de los mencionados conjuntos encontramos aspectos adquiridos espontáneamente o de forma automática, piénsese por ejemplo en los mecanismos perceptivos, y, por otro lado, elementos surgidos con otras características, especialmente los originados en el segundo y tercer eje. Estos harían referencia más al desarrollo cultural, pudiendo explicarse los mecanismos que los regulan a través de la ley de la doble formación de los instrumentos psicológicos superiores (Vygotsky, 1987). Procedemos ahora a exponer, sin ánimo de ser exhaustivos y con el sólo propósito de ilustración, cada uno de los conjuntos reseñados.

En primer lugar, desde la epistemología evolutiva se afirma que, de los tres posibles ámbitos en los que podemos dividir el mundo que nos rodea: el macronivel, el mesonivel y el micronivel, el sistema cognitivo humano está especialmente adaptado al meso nivel o nivel medio. Vollmer (1984) lo resume de la siguiente manera: "Cada organismo tiene su propio nicho cognitivo o ambiente, y asi es para el hombre. El nicho cognitivo del hombre nosotros lo llamamos mesocosmos. Nuestro mesocosmos es una sección del mundo real al que podemos hacer frente percibiendo y actuando, sensual y motoramente... El mesocosmos es hablando crudamente un mundo de medianas dimensiones...(p. 87)." “...nuestros órganos de los sentidos, la capacidad perceptual, las estructuras de vivencias, el lenguaje ordinario y los hábitos de inferencia elemental, están bien adaptados a este mesocosmos y son adecuados para las necesidades mesocósmicas. Lo mismo es cierto para nuestras formas de intuición. Nuestro poder de visualización está adaptado y adecuado a las necesidades cotidianas" (p. 88). Estos elementos componen un determinante de naturaleza bio-antropológica.

Un segundo conjunto lo integran los aspectos históricos. Nuestra capacidad para conservar en la memoria las experiencias pasadas y el conocimiento que 
nos llega de la narración histórica formal constituyen sus componentes esenciales. Estos nos dan cuenta tanto de los cambios que se han operado en las últimas décadas sobre multitud de aspectos relacionados con la concepción de la infancia, como de los trabajos de autores, fundamentalmente aquéllos que se han ocupado del conocimiento de la vida cotidiana (Aries, 1987), indicándonos que dicha concepción es, en cierto modo, subjetiva y en gran medida producto de una construcción sociocultural (Kagan, 1979, 1981; Yarrow, 1979, Kessen, 1979). Conceptos tan aparentemente decisivos como el papel de la madre en el desarrollo, la pasividad del niño en los primeros años,la infancia como período crítico, etc., se han ido viendo afectados tanto por las necesidades sociales que han ido apareciendo en los últimos años, piénsese en la incorporación de la mujer al trabajo, como por el avance que se ha experimentado en otros ámbitos. Todo ello ha contribuido a un cambio en muchos de los dominios aparentemente atemporales que se han terminado revelando como productos históricos perfectamente reemplazables. (Wertsch y Youniss, 1987. Young, 1990).

Por último, desde lo que hemos llamado el eje psicológico, encontramos que la disciplina formal a la que llamamos psicología ha tratado de poner de manifiesto las condiciones psicológicas a las que estamos apuntando. Por un lado aparecen en la Psicología Social dos grandes orientaciones. Ambas arrancan de los trabajos de principios de siglo sobre las actitudes y desembocan en la teoría de las representaciones sociales y en el cognitivismo social. Esta línea de evolución parte de los trabajos de Barlett (1932) sobre los factores sociales que moldean la memoria, sigue con los trabajos de Sheriff (1936) y Lewin (1935) y se prolongan en las aportaciones de Asch (1955) en torno a la formación de las impresiones. Las aportaciones de este último autor enlazan con las de Jerome Bruner sobre los procesos de categorización y sobre la tendencia que todos manifestamos de ir más allá de la información efectivamente proporcionada por la propia realidad para formarnos una imagen de la misma. (Bruner, 1957).

El eslabón que une estos primeros trabajos sobre las actitudes con los actuales sobre cognición social y representaciones, y que nos llevan al núcleo duro de lo que estamos exponiendo, es la obra seminal de Heider (1958). Este autor realiza una defensa de la importancia que reviste para la explicación psicosocial de las conductas el hecho de investigar seriamente en la psicología ingenua, refiriéndose con esto al sistema de conocimientos psicológicos, de sentido común, que utilizan las personas en su vida cotidiana, tanto para explicarse a sí mismas su propia conducta como para entender la de los demás y adecuar, en consecuencia, sus actuaciones.

Dentro de este marco nos vamos a centrar, por su importancia para lo que venimos comentando en la teoría atribucional y del aprendizaje social-cognitivo así como en los presupuestos que se defienden desde la teoría de las representaciones sociales. 
Para concluir, estos tres grandes ejes, o conjuntos de elementos explicitados, convergen, y en su caso hacen hincapié, en lo que podemos llamar el conocimiento personal, que es la base sobre la que se asientan, desde una perpectiva próxima y con un componente altísimo de carga psicológica, lo que denominamos concepciones.

\subsection{CONTRIBUCIONES AL ESTUDIO DE LAS CONCEPCIONES DESDE LAS TEORÍAS DE LA ATRIBUCIÓN Y DEL APRENDIZAJE SOCIAL-COGNITTVO}

Comenzaremos ocupándonos, en primer lugar, de las teorías atribucionales. La razón de incluir este posicionamiento en este punto está relacionada con el importante número de trabajos realizados desde esta perspectiva, su amplia fundamentación teórica y la validez ecológica de las investigaciones llevadas a cabo.

Bajo el rótulo de teoría de la atribución, diferentes investigadores han entendido cosas distintas. Algunos han llegado a plantear que no es sino un conjunto de cuestiones sacado de diferentes teorías. En general, se entiende por atribución los modos por los que explicamos y evaluamos tanto nuestra conducta como la de los demás. Se considera un elemento central en los procesos de interacción y relaciones entre personas proyectándose como una pieza clave de la cognición social. Uno de los objetivos que ha guiado la actividad científica en este campo ha sido el encontrar los procesos por los que se construyen dichas atribuciones.

Sea cual sea la perspectiva desde la que se realizan, las atribuciones que los profesores pueden hacer se dividen en tres categorías (Miller, 1995). Una primera hace referencia a lo inmediato. Esto es, a las causas o razones que los profesores afirman que hacen posible por qué el niño se comporta como lo hace. Podemos preguntar, por ejemplo, si una conducta es debida a rasgos disposicionales o situacionales, o si el éxito en una tarea es consecuencia de su habilidad o del esfuerzo realizado.

Un segundo tipo se dirige a los elementos que determinan la conducta a más largo plazo. El papel de los padres o profesores en el desarrollo o en qué medida es el niño producto de su código genético o del entorno son cuestiones que se abordan por los investigadores en este ámbito.

Un último tipo de atribución está integrado más que por factores causales específicos, tales como la habilidad o el esfuerzo, por dimensiones atribucionales más generales a lo largo de las cuales los factores específicos pueden clasificarse. Los trabajos de Weiner $(1985,1986)$, que identifica tres referentes, el "locus", la estabilidad y el control, son una prueba de este tercer tipo. Cualquier atribución referida a la conducta humana puede ser considerada a 
la luz de este modelo. La habilidad, por ejemplo, es generalmente vista como interna, en cuanto al "locus", estable a lo largo del tiempo, y no controlable; el esfuerzo, por el contrario, es entendido mayoritariamente como interno, inestable y controlable.

En cualquiera de los tres acercamientos la posibilidad de intercambiar el constructo atribución por el de creencias o concepciones podría estar plenamente justificada. Algo similar podemos extrapolar en el caso de las referencias que más tarde realizaremos con motivo de la teoría social-cognitiva.

Cobra especial relevancia en los estudios que estamos comentando los trabajos encaminados a desvelar los determinantes de la atribución. En un análisis pormenorizado podemos dividirlos en dos categorías: los que tienen que ver con las personas o conductas que están siendo enjuiciadas y los que influyen sobre las personas que están realizando el juicio atributivo.

Desde la perspectiva de la teoría social-cognitiva también se desprenden importantes cuestiones para la conceptualización que estamos llevando a cabo. Así, en el planteamiento que hasta aquí hemos venido haciendo de las concepciones, nos hemos referido a las consecuencias que tienen para los sujetos la manera en cómo son percibidos; en este apartado, sin abandonar totalmente aquella perspectiva, nos referiremos también a las consecuencias que para los propios sujetos tienen la forma en cómo ellos mismos se conciben. Trataremos de destacar el peso de diferentes factores personales en el proceso de la formación de las concepciones haciendo especial mención a sus consecuencias.

Una posición teórica y de investigación que ha provocado un fuerte impacto, tanto en el campo de la educación como en la manera de entender cualquier problema relacionado con el comportamiento humano, es la surgida del posicionamiento de Albert Bandura; especialmente, su postulado de la existencia, en cualquier sujeto, de un sistema autorregulatorio que le permite observar y evaluar su propio rendimiento. El juicio que la persona hace a partir de sus propias autoevaluaciones influye en las concepciones sobre su propia capacidad; es lo que Bandura (1982) llama autoeficacia.

Las cuatro fuentes a través de las cuales las personas pueden obtener información relevante sobre el sentido de autoeficacia son: su propio rendimiento, la experiencia vicaria, la persuasión verbal y los estados fisiológicos (Bandura y otros, 1986).

La primera fuente de información acerca de la autoeficacia es el resultado del rendimiento. El éxito alcanzado en una tarea dada enriquecerá el sentido de autoeficacia. La segunda fuente, la experiencia vicaria, funciona a través de la identificación del observador con un modelo competente. Si de esa identificación el observador obtiene la idea de que es igual o mejor que el modelo su percepción de autoeficacia aumentará. Ocurrirá lo contrario si 
la competencia del modelo es vista como distante o inalcanzable por el observador.

La persuasión verbal, tercera fuente de información, hace referencia a las influencias que otras personas desde el entorno, padres, iguales, etc, pueden ejercer utilizando los mensajes verbales para advertir o fomentar la capacidad y eficacia de una persona en un aspecto determinado. Aquí los otros ejercen la función del propio sujeto en el proceso de autoevaluación. La persuasión verbal puede manifestarse también a través del habla que el propio sujeto se dirige hacia él mismo. Para finalizar, los estados fisiológicos de la persona, última fuente, facilitan un conjunto de informaciones acerca de la autoeficacia. Hace referencia al estado de ánimo que se posee ante el convencimiento de poder conseguir el objetivo marcado.

El peso de la segunda y tercera fuente de información para la conformación de la creencia de autoeficacia es, en nuestro caso, de especial relevancia, ya que tienen que ver con las influencias que desde el entorno social favorecen y posibilitan determinadas construcciones en el desarrollo del niño.

Desde la teoría social cognitiva (Bandura, 1995) se propone un modelo que puede explicar cómo las concepciones pueden determinar importantes consecuencias para la conducta a través de un entrelazamiento de influencias.

Teniendo como marco lo que hasta ahora hemos venido apuntando, procederemos a continuación a conceptualizar las concepciones y su vinculación con el comportamiento humano en general y, más concretamente, con las ideas de los profesores.

\subsection{DEL ESTUDIO DE LAS CREENCIAS, IDEAS Y REPRESENTACIONES AL ESTUDIO DE LAS CONCEPCIONES}

Las concepciones son un constructo que los investigadores han creado para referirse a parte del conocimiento personal que los seres humanos poseen. En pie de igualdad con este término, ha aparecido en la literatura relacionada con el pensamiento de los padres, profesores, etc., un conjunto de expresiones que principalmente tienen en común con él su marcado carácter de conocimiento personal y a la vez social, así como su gran importancia de cara a los procesos de influencia educativa.

Con un sentido similar al que aquí le damos a la palabra concepción, han aparecido en los últimos años diferentes posicionamientos que incluían términos tales como representación, creencias, ideas, teorías personales, teorías intuitivas, actitud, perspectivas, etnoteorías etc. (Nespor, 1987). Entre todas las denominaciones utilizadas para etiquetar los trabajos destacan, por 
su frecuencia de aparición, el término creencia y el de idea para referirse al pensamiento de los profesores u otros elementos personales intervinientes en los sistemas educativos, y el de representación social con un carácter más genérico respecto de los contenidos de que se ocupan, es decir, sin centrarse tanto en cuestiones de naturaleza educativa como lo hacen en el caso de los dos primeros. El uso de dichos términos no es, sin embargo, ni consistente ni excluyente. En realidad nos los encontramos en bastantes estudios funcionando como sinónimos y mezclados a su vez con otras denominaciones. Conviene, no obstante, hacer algunas aclaraciones en un campo que se encuentra escaso de una teorización sólida.

El concepto de representación social, surgido del trabajo seminal de $\mathrm{S}$. Moscovici, La psychanalyse son image et son public, ha sido definido por Jodelet (1989) como: "Modalidades de pensamiento práctico orientadas hacia la comunicación, la comprensión y el dominio del entomo social ...". Presentan características sobre el plan de organización de los contenidos, operaciones mentales y de la lógica.

En todas las definiciones de representación social encontramos los siguientes elementos comunes:

- Son una forma de conocimiento social elaborado y compartido.

- Poseen una intencionalidad práctica.

- Pueden ser abordadas como productos pero también como procesos.

- Es necesaria la actividad de apropiación de la realidad social, más generalmente exterior al pensamiento así como la elaboración psicológica y social de esta realidad.

- Se sitúan estrechamente en las relaciones simbólicas inter e intragrupos.

El fuerte carácter constructivo de las representaciones sociales ha sido puesto de manifiesto de forma exhaustiva. Esto se puede abordar desde seis perspectivas distintas (Jodelet, 1984):

- El sujeto construye su representación según una dimensión de contexto y una dimensión de pertenencia. Desde esta perspectiva éstos son los aspectos puramente cognitivos.

- Un segundo enfoque pone el acento sobre los aspectos significantes de la actividad representativa. Al utilizar el individuo sistemas de codificación e interpretación proporcionados por la sociedad es por lo que esta representación es considerada como la expresión de dicha sociedad.

- Una tercera corriente trata la representación como una forma de discurso y desprende sus características de la práctica discursiva de sujetos socialmente situados. 
- La cuarta óptica la compone la práctica social del sujeto.

- Para el quinto punto de vista, el juego de las relaciones intergrupales determina la dinámica de las representaciones.

- Finalmente una última perspectiva basa la actividad representativa en la reproducción de los esquemas de pensamiento socialmente establecidos.

En resumen, la representación social según la perspectiva que se adopte puede ser exclusivamente individual (primera posición) o individual, integrando la inserción social del sujeto (los dos últimos apartados), o colectiva (posiciones segunda, tercera y cuarta).

Resulta evidente que las representaciones sociales trascienden la esfera de simples opiniones, imágenes y actitudes. Se trata de "sistemas cognitivos que poseen una lógica y un lenguaje particulares...de teorías, de ciencias sui generis, destinadas a descubrir la realidad y ordenarla" (Moscovici, 1983). Su función proviene de que son compartidas a nivel de una misma comunidad por lo que se refiere a las representaciones colectivas, como las religiones y los mitos, a cuyo estudio se dedicó Durkheim, oponiéndolas a las representaciones individuales que entran en el campo de la Psicología. Más sociales que éstas últimas que son manifestaciones puramente cognitivas, menos globales que los mitos y los fenómenos similares estudiados por antropólogos y sociólogos, las representaciones, en su actual concepción, permiten a los individuos orientarse en su entorno social y material, y dominarlo (Moscovici, 1983).

La teoría de las representaciones sociales, por su consistente fundamentación, refinamiento teórico, amén de por la vasta tarea de investigación que se ha venido realizando teniéndola como referente, constituye un sólido pilar de nuestro trabajo. No obstante, cabría destacar que su carácter excesivamente estructuralista, en cuanto a su concepción de lo personal, y su posicionamiento exageradamente descriptivo, ha evitado una identificación total con sus postulados. A pesar de ello reiteramos, una vez más, las amplias y esclarecedoras aportaciones que hemos encontrado tanto en sus supuestos teóricos como en los resultados de investigación surgidos a su sombra.

El término creencia es, sin lugar a dudas, el más frecuente entre los estudios realizados en este campo. Las creencias han sido conceptualizadas como el más sencillo e importante constructo en investigación educativa. (Fenstermacher, 1979). Pese a ello, ha tenido diferentes avatares y su uso ha sido un auténtico ejemplo de lo que no debiera hacerse en investigación.

Las conclusiones obtenidas en el estudio de revisión de Pajares (1992) nos informan de algunas características claves sobre el tema:

a) Las creencias se forman muy pronto y tienden a autoperpetuarse, perseverando incluso contra las contradicciones causadas por la razón, escolarización o experiencias. 
b) Los individuos desarrollan un sistema de creencias y lo adquieren a través del proceso de transmisión cultural.

c) El sistema de creencias tiene una función adaptativa, ayudando a los individuos a definir y comprender el mundo y a ellos mismos.

d) Las creencias sirven como filtro cognitivo.

e) Las creencias epistemológicas juegan un rol clave en la interpretación del conocimiento y monitorización cognitiva.

Pese a esta y a otras caracterizaciones que puedan hacerse, todos los trabajos realizados bajo este constructo, en la práctica, suelen reflejar un excesivo énfasis en los aspectos relacionados con los automatismos y la dimensión emocional-afectiva del comportamiento humano. Se observa, por lo general, que las creencias se sitúan al margen del control del sujeto que las posee, a pesar de ser individuales; los estudios nos las ofrecen, en la mayoría de los casos, como reglas estáticas e inalterables a las que el sujeto esta supeditado (como si de una ley física se tratara). Así pues, se destacan de este constructo su estabilidad, automaticidad, su vinculación con lo emocional y su marcado carácter idiosincrásico o personal.

El constructo ideas ha sido bastante menos manejado en la literatura, siendo más frecuente encontrarlo como sinónimo del concepto creencia, al menos en los textos que hemos manejado. En muy pocos trabajos, si exceptuamos los de Goodnow (1988) y Palacios (1987), aparece en el título el término ideas. De acuerdo con el último autor, cuando hablamos de ideas de los padres, nos referimos a percepciones, conocimientos, suposiciones e inferencias, valores o aspiraciones, expectativas, actitudes y atribuciones; además, a estos siete contenidos se deben añadir la capacidad percibida de influencia que los padres creen tener sobre el desarrollo y la educación de sus hijos, y las estrategias educativas preferidas por aquéllos en su relación con éstos. A esto se añadiría el grado de coherencia interna entre diversos contenidos, grado de flexibilidad, carácter más o menos estereotipado, status más o menos explícito y mayor o menor abstracción.

El planteamiento reseñado representa bastante bien lo que este tipo de estudios suele entender que hay bajo el rótulo ideas. Nos interesa, ante todo, destacar su marcado acento en los aspectos cognitivos del comportamiento, haciendo caso omiso de lo afectivo-emocional. En cierto sentido, podemos decir que este acercamiento está interesado en el procesamiento de informaciones del sujeto aislado, sin reparar, por ejemplo, en los procesos perceptivos previos al propio procesamiento. Responde a lo que en palabras de Bruner (1985) se denomina un "conocedor paradigmático".

Para concluir este apartado, proponemos que todo el campo del que se tratará en este estudio sea etiquetado con el término concepción. Entendemos que con el sentido que le damos aquí, ha sido utilizado muy pocas veces, al menos 
en el campo de la literatura referida a profesores, y aún menos en relación con la forma de concebir la evaluación. Pero no queremos adelantar lo que será nuestra propuesta sobre las concepciones que realizaremos en apartados posteriores.

\subsection{DE LAS CONCEPCIONES A LOS COMPORTAMIENTOS}

La literatura que hemos seguido para cumplimentar este apartado está integrada por estudios que se han centrado especialmente en las concepciones de los profesores. No debe olvidarse, sin embargo, que el problema desborda el ámbito en el que nos encontramos; tratar de darle solución aquí sería un intento tan vano como pretencioso.

En general, se puede decir que el interés de los que se han ocupado de esta cuestión es demostrar que las conductas de los profesores, cuando participan en los procesos de evaluación, están mediadas por sus propias concepciones sobre este aspecto del currículum. Algunas investigaciones han mostrado que estas relaciones no son significativas, aunque son muchos más los estudios que muestran resultados positivos. En general podemos decir que aunque exista relación, ésta no es ni lineal ni mecánica. Sigel (1986) propone la "metáfora del embudo", según la cual los profesores, padres y demás adultos son comparables a un embudo en el que dejamos caer una serie de ideas siendo el resultado un escaso número de acciones. Así la acción no es el efecto lineal de dichas ideas, sino el producto de una combinación de ellas.

En cualquier caso, la forma en cómo se plasman las ideas en la conducta dependerá de diferentes factores moduladores que están relacionados tanto con los profesores y sus características, como con la materia de que se trate, los alumnos y, por último, con los contextos.

Para concluir, siguiendo a Palacios (1987), las concepciones de los profesores se expresan no sólo en conductas dirigidas directamente hacia las actividades de evaluación, sino también en todas aquéllas que tienen que ver con la forma de estructurar el proceso instructivo dentro de la propia aula.

En otro orden de cosas, es probable, no obstante, que el problema a plantear deba abordarse desde otras perspectivas. Nos referimos a que habitualmente se piensa en una relación lineal y jerárquica que arranca de las concepciones y termina en el comportamiento. Esta forma de entender la cuestión puede que esté ocultando unos procesos de relación mucho menos lineales y más dialécticos. Plantear que las acciones están mediadas por las concepciones es tan lícito como hacerlo al contrario.

Con lo que hasta ahora llevamos planteado, creemos que podemos hacer una propuesta. Ésta debe permitirnos caminar desde la perspectiva de los estudios sobre representaciones sociales, pasando por los de creencias y los 
que utilizan el referente de las ideas, para llegar a admitir la conveniencia de que se orienten hacia las concepciones, tal y como aquí las entendemos.

Las razones que tenemos son, en primer lugar, de carácter terminológico. Es deseable que se pueda llegar a un término, o denominación, claramente definido que permita utilizar el lenguaje con precisión. En este mismo trabajo se pueden ver referencias de investigaciones que usan de forma indistinta términos tales como creencia, ideas, etc, con un sentido totalmente impreciso propio del lenguaje coloquial. En realidad, hubiéramos tenido problemas para llevar a buen fin la realización de este trabajo, de habernos limitado a usar informes de investigación a los que le exigiéramos la precisión que estamos reclamando.

Añadido a los argumentos que ya se han manejado, hay un referente en el término concepción, siguiendo al grupo de la Universidad de Göteborg, que no lo encontramos en ninguno de los constructos utilizados habitualmente. Nos referimos a que la concepción no sólo apunta a lo que pensamos sobre el mundo, sino también a cómo lo pensamos. En consecuencia, cuando hablamos de concepción, estamos haciendo referencia también a una dimensión epistemológica del conocer que, como ya hemos apuntado en los apartados anteriores, es de suma importancia para entender el comportamiento más "humano" del hombre (Carmona 1995).

Un último conjunto de razones tiene que ver con la necesidad de utilizar conceptos que recojan, de forma simplificada y holística, todo el sentido que encontramos en los contenidos a los que nos referimos. Ya hemos visto que el término creencias está cargado de excesivos referentes afectivo-emocionales, inalterabilidad y falta de perspectivismo, que nos hablan de un sujeto que actúa automáticamente. El término ideas, por otro lado, apunta a referentes de naturaleza cognitiva que se ejecutan en el sistema de un sujeto aislado, situando en un segundo plano lo afectivo-emocional y lo socio-cultural o contextual. Nuestra propuesta entiende que para abarcar todos los elementos que están en juego: afectivo-emocionales, cognitivos y socio-culturales o contextuales, el término concepción puede servir como un elemento que los aglutine e integre.

El planteamiento que aquí seguimos es: Las concepciones, tal y como nosotros las entendemos, son un constructo de naturaleza psicosocial que nos permite entender y explicar el fenómeno de "ver", "pensar" y "sentir" el mundo que nos rodea de forma simultánea. Se trataría de integrar en un único elemento a estos complejos referentes.

En el primero de ellos, al que nosotros hemos denominado "ver", se incluye lo que está más volcado hacia lo estructural: histórico-cultural y contextual, de naturaleza fuertemente social y situada. Este primer apoyo de la conceptualización de las concepciones podría estar representado por el espectro que recoge la teoría de las representaciones sociales. El interés, no único 
pero si fundamental, de esta perpectiva radica en destacar lo socio-estructural, con su fuerte peso en el comportamiento, pero resulta reduccionista, desde nuestra posición al menos, entender que ésta es la única vía explicativa que tenemos. La teoría de las representaciones sociales, como contenidos y como teoría, nos permiten "ver" el mundo, y eso es necesario, pero necesitamos ir más allá para poder explicar el proceso de personalizar esa visión proporcionada por lo social y cultural. Anteriormente, cuando nos ocupamos de las representaciones, ya señalábamos ciertas debilidades en este mismo sentido que no vamos a repetir.

Frente al automatismo forzado por lo estructural que nos conduciría a una "visión" inevitable del mundo, destacado por la teoría de las representaciones sociales, encontramos el proceso autodirigido por el propio individuo que se empeña en "pensar" el mundo, bien es verdad que al principio ese mundo le viene dado, pero el sujeto va más allá de esto construyendo, o coconstruyendo con otros, distintas visiones. Este segundo elemento que nosotros integramos en la conceptualización que estamos realizando de las concepciones viene a estar representado por todos los planteamientos realizados al amparo del término ideas, conceptos, teorías, etc. En este caso todos los constructos se conciben como elementos que confieren sentido al mundo percibido. "Pensar", en este caso, lo interpretamos como dar sentido a lo percibido (Vygotsky, 1978). Esto no es más que encontrar el fin hacia el que se dirige la actividad humana o, más aún: la conciencia de la intención con que se lleva a cabo dicha actividad. En consecuencia, lo que llamamos concepciones encierra en su seno este segundo aspecto al que llamamos "pensar" que, en último término, apunta a un factor con un fuerte aspecto directivo sobre la conducta.

Por último, el tercer apoyo lo recibiría de la literatura desarrollada bajo la terminología de creencias. Aquí se destaca lo más propiamente afectivo-emocional con un fuerte carácter de automatismo. Es lo que podemos llamar la función valorativa de la conducta humana que está muy presente en el constructo concepción; nos referimos a los aspectos relacionados con el mundo del afecto y el sentimiento. Toda la investigación realizada al amparo de lo que se ha venido en llamar creencias tiene una impronta que lo relaciona más con el "sentir" que con cualquier otra dimensión del comportamiento. Su debilidad, para entenderlo como un único componente explicativo del compartimiento humano, se sitúa en un sentido similar al que señalábamos para las representaciones: marcado carácter estructural y automático; sólo que aquí, en vez de tener ese fuerte marcaje social, responde a un referente profundamente subjetivo y personal de naturaleza afectiva y emocional.

Para concluir, necesitamos integrar en un mismo ámbito lo que hay de socio-contextual, personal y afectivo-emocional en el comportamiento, y para hacerlo entendemos que integrar los tres elementos reseñados: representaciones, ideas y creencias en un sólo constructo, al que hemos denominado concepciones, es una propuesta que nos parece de sumo interés. En este sentido 
venimos a coincidir con el planteamiento que afirma que, cuando hablamos de concepciones, las entendemos como una estructura mental de carácter general que incluye creencias, conceptos, significados, reglas, imágenes mentales y preferencias, conscientes e inconscientes, que tiene su origen en la cultura del contexto y se desarrolla, en gran medida, gracias a los procesos personales.

\section{OBJETIVOS DE LA INVESTIGACIÓN}

Pretendemos conocer las concepciones de los profesores de Educación Secundaria sobre:

1. Qué evaluar.

2. Para qué evaluar.

3. Cuándo se debe evaluar.

4. Cómo se debe evaluar.

5. Cómo creen que se puede mejorar.

6. Qué es la evaluación.

\section{DESCRIPCIÓN DE LA MUESTRA}

Todos los profesores de Educación Secundaria de Granada de Centros públicos y privados constituyen la población de referencia a la que se desea generalizar los resultados.

Para realizar las entrevistas fue necesario extraer un subconjunto de profesores, de forma aleatoria, por razones de economía y tiempo, dada la gran cantidad de información que se acumula con las entrevistas y la necesidad de personal que exige la recogida, transcripción, codificación y análisis de dicha información.

En la selección se tuvieron en cuenta todos los Centros de Secundaria y, al azar, se seleccionaron dos profesores por Centro. En algunos casos no fue posible entrevistar a los dos, por negativa del profesor o por su no presencia en el Centro el día de la entrevista, por lo que de algunos Centros sólo tenemos un profesor.

Así pues, la muestra la formaron cincuenta profesores de Educación Secundaria, de edades comprendidas entre 28 y 54 años. 
De los 50 profesores entrevistados 31 fueron varones y 19 mujeres.

Impartían docencia en diferentes áreas, que agrupamos para fines de análisis e interpretación de la siguiente manera:

Ciencias Experimentales 15

Matemáticas y Lenguas 20

Sociales y otras áreas 15

Cada entrevista ocupó aproximadamente 10 minutos de grabación; así pues, se transcribieron y analizaron ocho horas de entrevistas, lo que supone una gran cantidad de información para el objetivo propuesto.

\section{PROCESO SEGUIDO EN LA RECOGIDA DE INFORMACIONES POR LAS ENTREVISTAS}

En este apartado expondremos: cómo hemos recogido los datos, cómo los hemos organizado y cuál ha sido el soporte informático que hemos utilizado.

Hemos elegido la entrevista semiestructurada porque nos permite, por una parte, recoger todos los aspectos que interesa conocer y, por otra, nos da la suficiente flexibilidad como para que el sujeto entrevistado exprese con libertad su opinión y nos permita acceder a sus creencias.

La entrevista se realizó a cada uno de los profesores tras fijar con ellos la fecha y hora. Para que los datos fueran recogidos de la forma más fidedigna posible todas las entrevistas se grabaron.

La entrevista que elaboramos seguía este guión básico:

1. ¿Qué considera que se debe evaluar en los alumnos?

2. ¿Qué finalidad cree que debería tener la evaluación

3. ¿En qué momento o momentos considera que se debe evaluar?

4. ¿Cómo considera que se debe evaluar? ¿Qué procedimientos o instrumentos considera más adecuados?

5. ¿Qué cree Vd. que necesita para mejorar su práctica de evaluación?

6. ¿Qué es para Vd. evaluar? 


\section{TRATAMIENTO CUALITATIVO DE LOS DATOS}

Una vez realizadas la entrevistas con grabadora, se procedió a su transcripción para pasar a su lectura y a tratar los datos.

La limitada capacidad humana para procesar grandes cantidades de datos hace necesario reducir la amplia información contenida en la datos textuales, diferenciando las distintas unidades a las que, posteriormente, daremos significado.

Plantear un análisis cualitativo implica seguir un proceso, más o menos estructurado y de carácter cíclico. En el proceso se siguen tres fases distintas que se pueden superponer en cualquier momento:

\section{A) SEPARACIÓN DE ELEMENTOS}

De acuerdo con el significado básico del término análisis, una de las primeras operaciones a las que sometimos la información fue la separación de los segmentos o unidades que conforman el conjunto global de datos objeto de análisis. Esta división en unidades relevantes y significativas es una de las premisas más características del análisis de datos cualitativos.

La división de la información en unidades puede realizarse siguiendo diferentes criterios:

1. Las consideraciones espaciales o temporales permiten definir unidades de carácter artificial, surgidas a partir de criterios exclusivamente físicos. De acuerdo con tales criterios, constituyen unidades los bloques de un determinado numero de líneas, las páginas, los bloques de minutos etc.

2. En función del tema abordado, según se consideren conversaciones, sucesos, o actividades que ocurren en la situación observada. Es posible encontrar segmentos que hablen de un mismo tema.

3. Criterios gramaticales o criterios conversacionales. En los primeros, el párrafo o la oración son considerados las unidades básicas del texto; en los segundós, se divide teniendo en cuenta las declaraciones, o los turnos de palabra. En ambos casos, no es necesario un juicio sobre el significado para establecer los límites de la unidad.

En nuestro análisis hemos utilizado la opción b: división de la información en unidades en función del tema abordado. 


\section{B) IDENTIFICACIÓN Y CLASIFICACIÓN DE ELEMENTOS}

Con la identificación y clasificación de elementos examinamos las unidades de datos (segmentos) para encontrar en ellas determinados componentes temáticos que nos permitan clasificarlas en una u otra categoría de contenido. Las operaciones más representativas de las actividades de identificación y clasificación son las conocidas como codificación y categorización. La categorización hace posible clasificar las unidades que son cubiertas por un mismo tópico. Las categorías pueden referirse a situaciones y contextos, actividades y acontecimientos, relaciones entre personas, comportamientos, opiniones, perspectivas sobre un problema, métodos y estrategias, procesos. La categorización se realiza conjuntamente con la segmentación cuando ésta se hace atendiendo a criterios temáticos.

El proceso de codificación no es más que la operación concreta por la que asignamos a cada unidad un indicativo (código) propio de la categoría en la que la consideramos incluida. En el proceso físico, se asigna una etiqueta a la categoría. El estudio de ambas operaciones se suele hacer en investigación educativa de forma conjunta, incluso en algún momento llegan a identificarse como un único proceso.

Los códigos, que represèntan a las categorías, pueden ser números, aunque nosotros hemos seguido la costumbre, más frecuente, de utilizar abreviaturas de palabras con las que se designan las categorías. Además, utilizar nombres es más eficaz, ya que los relacionamos estrechamente con el concepto original al que nos referimos.

Las categorías utilizadas en un estudio pueden establecerse a priori, partiendo: de un marco teórico y conceptual previo, de cuestiones o hipótesis que guíen una investigación, de categorías ya usadas en estudios de otros investigadores, o de los instrumentos de investigación empleados (el uso de cuestionarios o guiones de entrevista, por ejemplo, permite utilizar las cuestiones planteadas como fuente de categorías útiles para organizar los datos).

El partir de un sistema de categorías a priori no es una condición suficiente, sino un requisito previo para conseguir una misma codificación en distintos codificadores. Es preciso además una definición operativa de categorías, ofreciendo reglas que especifiquen los aspectos del contenido que deben tomarse como criterio de pertenencia a una categoría.

Pero los sistemas de categorías también pueden elaborarse inductivamente a partir de los propios datos. Al examinar los datos nos preguntamos por el tópico capaz de dar significado a cada segmento. Posteriores comparaciones entre los datos agrupados bajo un mismo tópico, o entre tópicos, permitirán refinar las categorías emergentes. 
Esta segunda posibilidad es la que hemos decidido utilizar en nuestro estudio, porque nos permite acercarnos al análisis de los datos de forma más libre y, en posteriores fases de la investigación, compararlas con sistemas de categorías ya establecidas.

Debe quedar claro que tanto si los códigos hubieran sido preelaborados en función del estudio, o definidos a partir de los datos, como hemos procedido nosotros, la codificación y categorización no se desarrollan linealmente, sino que, al ser procesos recurrentes, se pueden ir alterando continuamente. En la medida en que codificamos, vimos que las categorías, desarrolladas en principio, no eran siempre adecuadas. Muchas veces hemos tenido que renombrarlas y modificarlas en su contenido o incluso sustituirlas por otras nuevas.

Para darle consistencia a las categorías hemos procurado cumplir algunos requisitos de un sistema de categorías (Anguera 1995, Buendía, en prensa) tales como: exhaustividad, pertenencia, objetividad, etc.

\section{C) AGRUPAMIENTO}

La identificación y clasificación de elementos están estrechamente unidas al agrupamiento. Cuando categorizamos estamos ubicando diferentes unidades de datos bajo un mismo tópico o concepto teórico. La categorización supone en sí misma una operación conceptual de agrupamiento.

En nuestro caso, al partir de seis cuestiones centrales, el agrupamiento se ha realizado en función de las preguntas.

En conclusión, las tareas revisadas hasta aquí (segmentación en unidades, codificación/categorización, agrupamiento) constituyen modos de contribuir a la reducción de datos, que en el fondo presupone todo análisis (se parte de un conjunto amplio y complejo de datos hasta llegar a elementos más manejables que permitan establecer relaciones y sacar conclusiones).

\section{ANÁLISIS DE LAS ENTREVISTAS: AQUAD V.3.0}

El análisis cualitativo de los datos implica un proceso complejo en el que se trabaja con gran cantidad de datos que tenemos que reducir. La ayuda de la informática en este proceso es esencial. De los programas existentes en el mercado nos decidimos por el AQD. El programa AQUAD o AQD (Analysis of Qualitative Data) ha sido elaborado a partir del sistema de manejo de datos cualitativos Qualog de Shelly, adaptado al entorno del Ordenador Personal, mediante un lenguaje de Inteligencia Artificial Turbo-Prolog. 
A continuación hacemos el estudio de las respuestas dadas por los profesores a cada una de las seis cuestiones que se le realizaron para conocer sus creencias sobre aspectos de la evaluación.

\section{¿QUÉ CREE QUE SE DEBE EVALUAR EN LOS ALUMNOS?}

Las respuestas dadas a esta cuestión han quedado recogidas en nueve tópicos: conocimiento, habilidades, actitudes, procedimientos, aptitudes, factores personales, valores, capacidad crítica y expresión. Gráficamente, las distintas categorías establecidas por los profesores de manera global quedan así:

\section{¿Qué cree que se debe evaluar?}

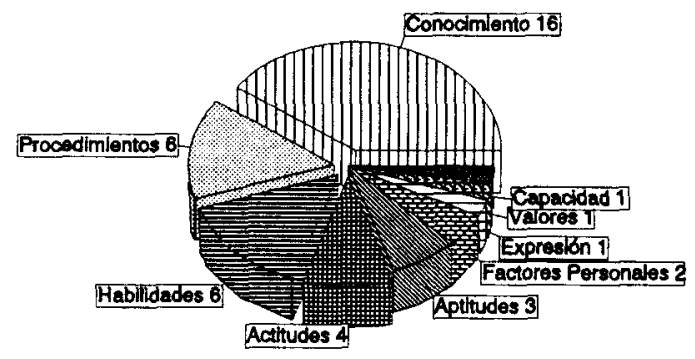

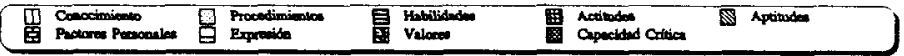

GRÁFICO 1

Indudablemente, el dato más destacable es la referencia que hacen los profesores a la evaluación de los conocimientos de sus alumnos. De todos los elementos que los profesores dicen que se deben evaluar, el conocimiento es elegido por el $95 \%$ de los profesores que componen la muestra. Esta práctica unanimidad disminuye a la hora de determinar el tipo de conocimiento que se tiene que evaluar. Para algunos profesores, el conocimiento no debe ser memorístico. Tampoco parece haber acuerdo respecto a qué valor se le debe dar respecto a los otros elementos.

Procedimientos, habilidades, actitudes y aptitudes también son categorías a las que se le da bastante importancia a la hora de evaluar, aunque los profesores manifiestan ciertas reticencias a hacerlo, entre otras causas debido a que no existen suficientes instrumentos elaborados para ello. 


\section{¿QUÉ FINALIDAD CREE QUE DEBERÍA TENER LA EVALUACIÓN DE LOS ALUMNOS?}

La cuestión genera una serie de respuestas que corroboran las concepciones que tradicionalmente se atribuyen a las finalidades de la evaluación.

Una evaluación puede tener dos fines: el eminentemente formativo y el de promoción o no del alumno.

\section{¿Qué finalidad cree que tiene la evaluación?}

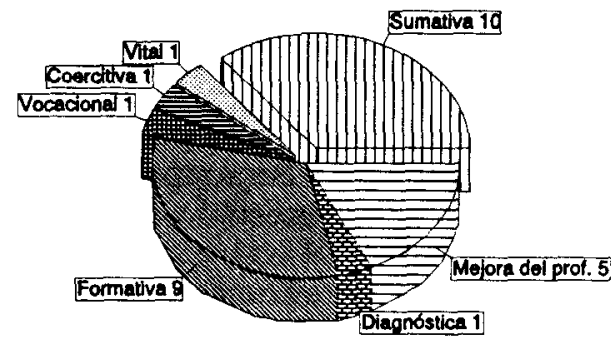

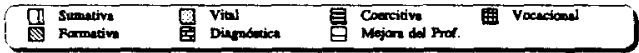

\section{GRÁFICO 2}

En el sector circular se puede ver la frecuencia de las respuestas de los sujetos entrevistados, que quedan estructuradas en dos categorías. La primera engloba seis términos referidos a la finalidad que se sigue respecto a los alumnos (sumativa, formativa, coercitiva, vocacional, vital, diagnóstica); la segunda referida al propio profesorado: finalidad de perfeccionamiento y mejora de la docencia.

Como decíamos, la finalidad que este grupo de profesores cree que tiene la evaluación es para conocer si tienen los conocimientos suficientes para superar la materia, finalidad sancionadora, y que los legitima para pasar al siguiente nivel, sin olvidar el carácter formativo que también consideran que debe ser contemplado.

Es de destacar que un importante porcentaje de profesores cree que la evaluación de los alumnos debe suponer para el profesor un perfeccionamiento, una mejora para el mismo; incluso en algunos casos se propone una evalua- 
ción de los alumnos al profesor. Aparecen además, tres categorías, aunque de forma un poco residual: la finalidad coercitiva, la vocacional y la vital. La primera viene a poner de manifiesto el poder de intimidación mediante el uso de las calificaciones; el profesor decide quién aprueba y quién suspende.

La categoría vocacional hace referencia a que la evaluación puede servir para que el estudiante descubra si realmente desea estudiar o, por el contrario, incorporarse al mundo del trabajo.

La categoría vital viene a destacar la capacidad de la evaluación para hacer que el alumno se dé cuenta de que no sólo es necesario tener conocimientos sino que, además, se necesita saber que uno vive en una comunidad en la que debe participar y conocer el lugar que ocupa.

\section{¿EN QUÉ MOMENTOS CREE QUE SE DEBE EVALUAR?}

Los profesores creen que la evaluación se puede realizar en tres momentos distintos: en un primer momento y como toma de contacto, a lo largo de todo el proceso y al finalizar dicho proceso.

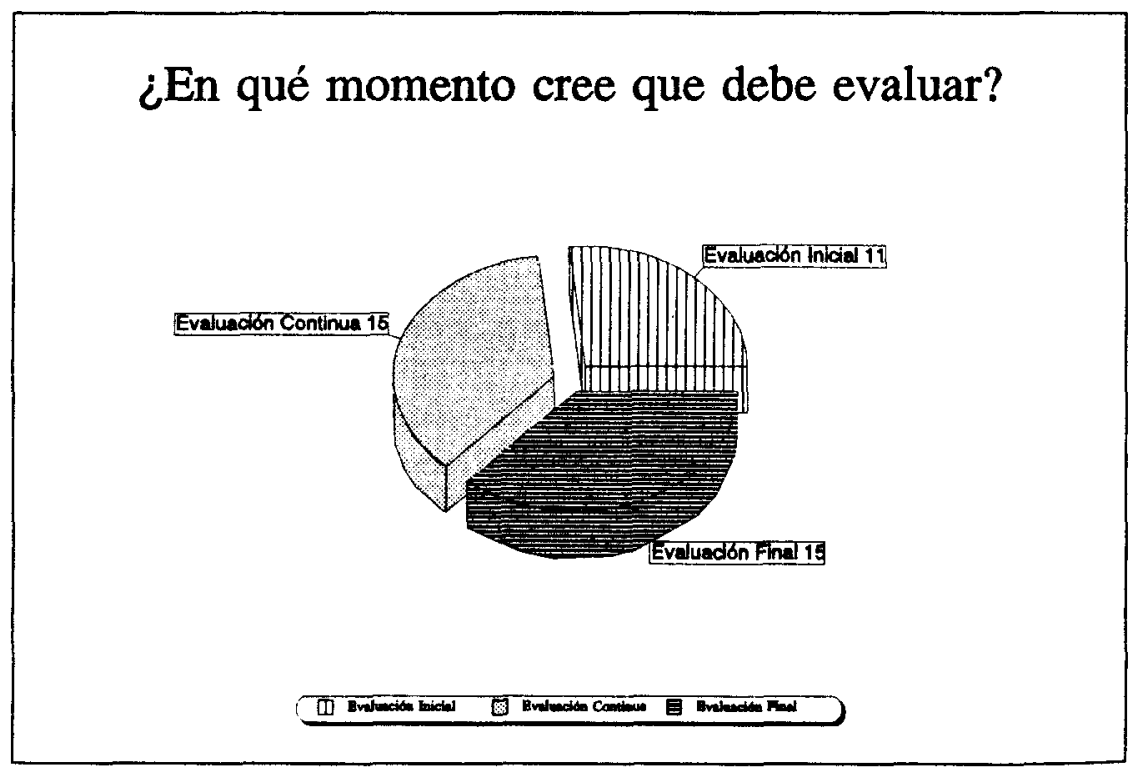

GRÁFICO 3

El conjunto de los entrevistados cree que los tres momentos evaluativos son necesarios en el ámbito educativo. Los profesores creen en el carácter diagnóstico de la evaluación inicial, pero tienen ciertas reticencias a realizarla debido a su creencia sobre el proceso de enseñanza-aprendizaje. Es 
interesante destacar que consideran innecesaria la evaluación inicial por la disparidad de los sujetos que hay en el aula (heterogeneidad de las clases). El nivel es tan diferente que, aunque diagnosticaran cuál es la situación de partida de sus alumnos, les sería imposible adaptar su enseñanza a tal variedad.

La segunda cuestión está relacionada con el número de alumnos que hay en clase y que impide poder realizar pruebas iniciales con carácter diagnóstico.

La evaluación continua y la final son las que los profesores ven más necesarias, dándole a la última un carácter marcadamente sumativo, al final del curso, con vistas a la promoción. La continua es considerada de gran importancia en el proceso evaluativo.

\section{¿CÓMO CREE QUE SE DEBE EVALUAR A LOS ALUMNOS? ¿QUÉ TÉCNICAS E INSTRUMENTOS CREE QUE SE DEBEN UTILIZAR?}

De forma global, las categorías que establecemos en este apartado se pueden agrupar de acuerdo con dos tópicos. El primero estaría relacionado con instrumentos que el profesor cree que es conveniente utilizar en la evaluación de sus alumnos, y el segundo con procedimientos de evaluación.

En el primer apartado, instrumentos, hemos incluido: trabajos, test, preguntas a desarrollar y examen oral. En los procedimientos incluimos: el diálogo, la observación, la autoevaluación y los seminarios.

Partimos del principio de que los profesores consideran que los instrumentos que necesitan y su forma de evaluar depende de las características específicas de la asignatura de la que estamos tratando. No obstante, consideran, desde su experiencia, que existen algunos instrumentos o técnicas más adecuados para la evaluación. Aparecen en el siguiente gráfico. 


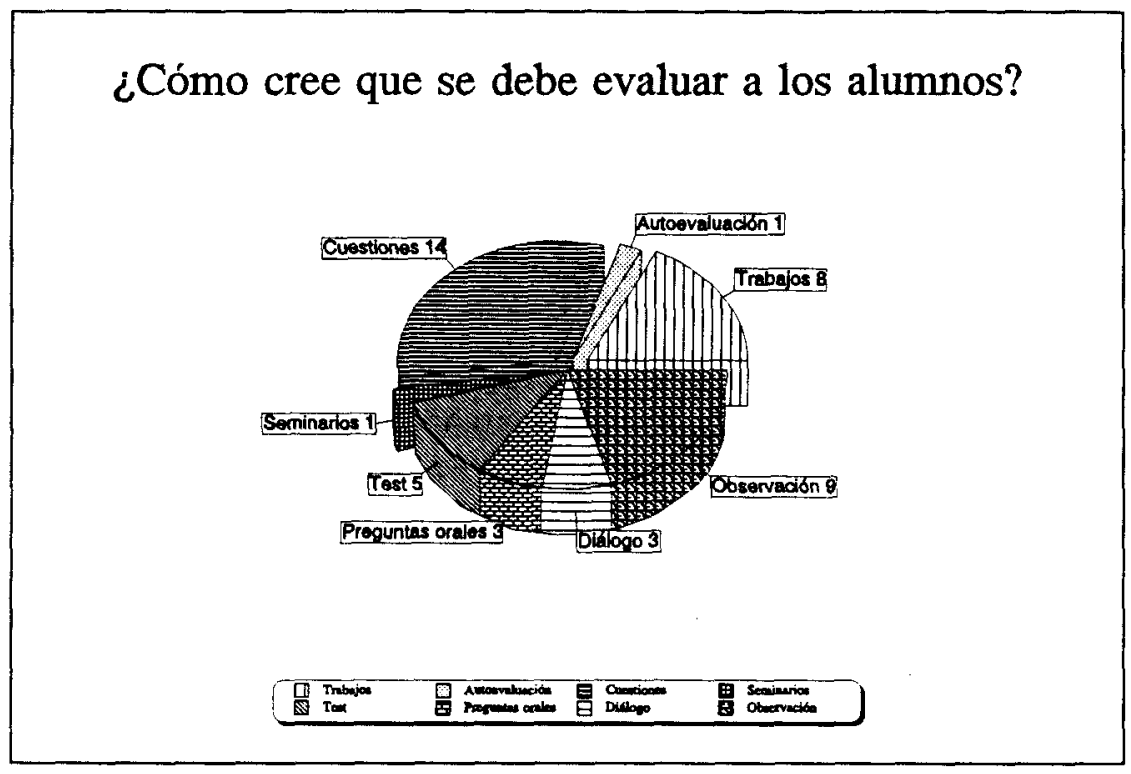

GRÁFICO 4

Los trabajos, tanto de clase como los realizados en las casas, creen los profesores que serían buenos indicadores para realizar la evaluación, tanto si están realizados individualmente como en grupo. En la mayoría de los casos son considerados elementos complementarios de otras formas de evaluación, como por ejemplo los exámenes tradicionales. Las preguntas de desarrollo creen los profesores que es la forma más habitual de exámenes escritos.

Es de destacar la observación como técnica para realizar la evaluación. Consideran que se debe de utilizar, aunque no ha habido unanimidad en la respuesta. Aunque raramente suele ser el único elemento de evaluación, sí se convierte en un elemento que complementa a la evaluación que se realiza con el tradicional examen escrito. Destaca como elemento innovador las creencias sobre la importancia del diálogo (que no es un examen oral, sino un proceso de intercambio entre el profesor y el alumno), la autoevaluación y la utilización de seminarios para evaluar.

Los profesores, independientemente de la materia que imparten, siguen creyendo que, en las circunstancias en las que se desarrolla su enseñanza, el mejor método para evaluar el progreso de los alumnos es la utilización del tradicional examen escrito con cuestiones de desarrollo más o menos largas. 


\section{¿QUÉ CREE QUE SE NECESITA PARA HACER UNA MEJOR EVALUACIÓN DE LOS ALUMNOS?}

Las necesidades que perciben los profesores de Educación Secundaria para realizar una mejor evaluación de sus alumnos son muy diversas. Podemos agruparlas en éstas: mayor reflexión del profesorado, mayor organización de las asignaturas, formación del profesorado en temas específicos de evaluación, diálogo entre profesores. El otro aspecto estaría relacionado con elementos administrativos: menor número de alumnos, mayor cantidad de libros, posibilidad de dedicación de un número de horas mayor a los alumnos en tutoría, mayor espacio y más profesorado.

Los datos globales quedarían estructurados de la siguiente forma:

\section{¿Qué cree que necesita para evaluar...}
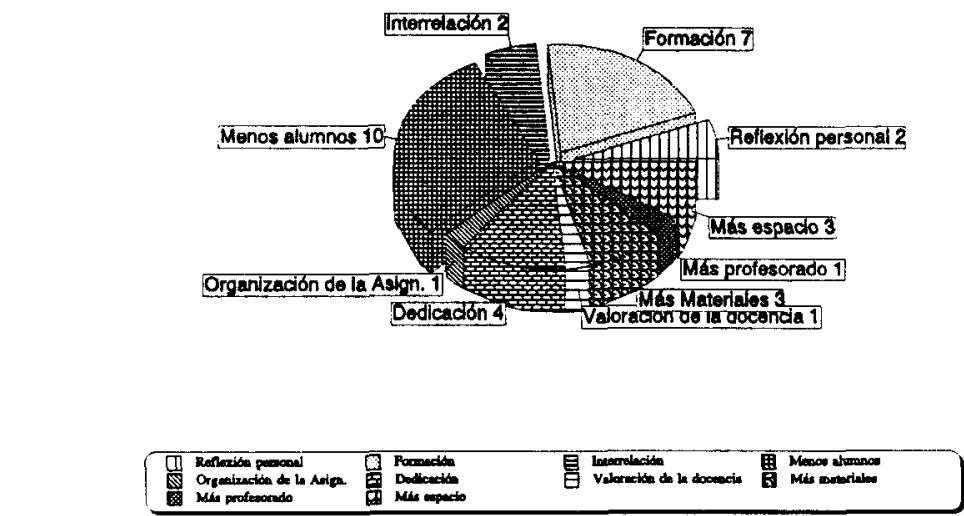

GráfICO 5

Los profesores creen que mejoraría la evaluación con cursos de formación sobre diversos aspectos: adecuación a las nuevas necesidades evaluativas e instrumentos de evaluación.

También creen que necesitan más tiempo para dedicárselo a su alumno para lo que sería necesario descargar su número de horas de docencia por horas de tutoría, en las que la atención individualizada al alumnos es mucho mayor.

Los tres grupos de profesores, muestran acuerdo en los aspectos relacionados con un menor número de alumnos por clase y la necesidad que existe 
de materiales adecuados, principalmente libros. También parece existir acuerdo en la creencia de que una mejor evaluación implica una formación del profesorado más adecuada.

\section{¿QUÉ CREE QUE ES EVALUAR?}

La evaluación es un concepto que, según los sujetos entrevistados, viene caracterizada por una serie de términos. Los que han presentado una mayor frecuencia son: valorar, sancionar, observar, seguir el proceso de construcción de conocimiento del alumno.

\section{¿Qué cree que es evaluar?}

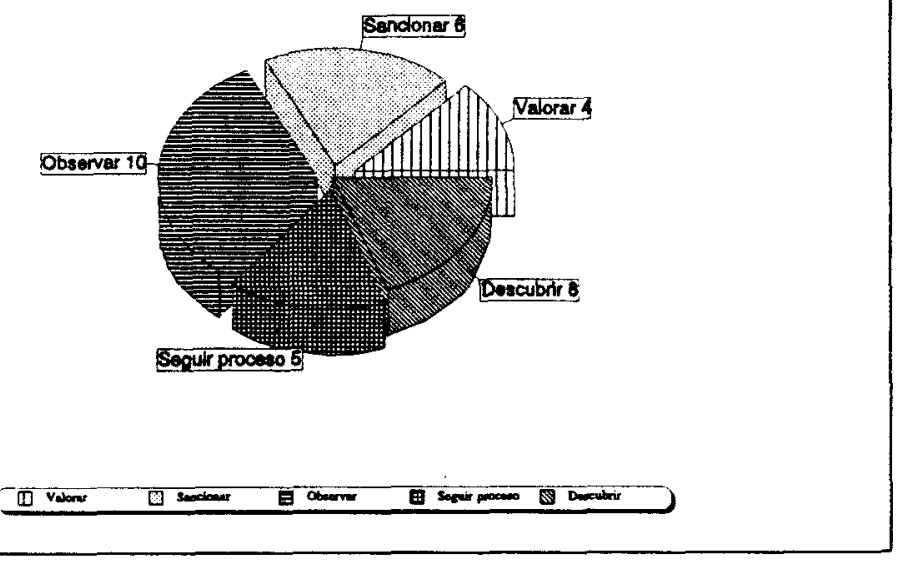

\section{GRÁfico 6}

Podemos decir que la definición de cada sujeto es distinta, propia y específica de cada uno y, a la vez, con elementos comunes en todos ellos. Una vez analizadas todas ellas y recogiendo los términos que aparecen en el gráfico, podemos resumir que, para los profesores entrevistados, la evaluación es un proceso que pretende observar tanto a los alumnos como al Centro y a los profesores, para mejorar el sistema a través de ella y, a la vez, promocionar a los que mejor han conseguido las metas que el profesor propone.

\section{CONCLUSIONES}

Estas conclusiones están referidas a las concepciones que los profesores de Educación Secundaria tienen sobre la evaluación 
1. Las concepciones sobre qué evaluar las hemos agrupado en nueve categorías: conocimientos, habilidades, actitudes, valores, procedimientos, aptitudes, factores personales, capacidad crítica y expresión.

De todas ellas, el conocimiento, como principal objeto de evaluación ha sido considerado por el $99 \%$ de los profesores que componen la muestra.

2. La finalidad que este grupo de profesores cree que tiene la evaluación es la de conocer si el alumno ha conseguido los conocimientos suficientes para superar la materia y promocionar al siguiente nivel, sin olvidar el carácter formativo que también consideran que debe ser contemplado.

3. La evaluación continua y la final son las que los profesores consideran más necesarias, dándole a la última un carácter marcadamente sumativo, al final del curso, con vistas a la promoción.

Es interesante destacar que consideran necesaria la evaluación inicial, pero creen que, no es efectiva por lo heterogéneas que son las clases. El nivel es tan diferente que aunque diagnosticaran cuál es la situación de partida de sus alumnos, les sería imposible adaptar su enseñanza a tal variedad.

4. Los profesores consideran que los instrumentos que necesitan y su forma de evaluar dependen de las características específicas de la asignatura que evalúan, pero en ningún momento hacen referencia a las características personales del alumno, ni al proceso de enseñanza-aprendizaje, ni a las finalidades que se persiguen, etc. No obstante, consideran, desde su experiencia, que existen instrumentos o procedimientos más adecuados para la evaluación. Creen necesario el examen o prueba objetiva.

5. Las necesidades que perciben los profesores de Educación Secundaria para realizar una mejor evaluación de los alumnos son muy diversas. Podemos resumirlas en dos grupos:

El primero relacionado con el propio profesor: mayor reflexión del profesorado, mayor organización de las asignaturas, formación del profesorado en temas específicos de evaluación, diálogo entre profesores.

El segundo relacionado con elementos administrativos: menor número de alumnos, mayor cantidad de libros, posibilidad de dedicación de un número de horas mayor a los alumnos en tutoría, mayor espacio y más profesorado.

Los profesores creen que mejoraría la evaluación con cursos de formación sobre diversos aspectos, fundamentalmente: adecuación a las nuevas necesidades evaluativas e instrumentos de evaluación.

6. Respecto al concepto de evaluación, para los profesores de la muestra es un proceso que pretende observar tanto a los alumnos como al Centro 
y a los profesores, para mejorar el sistema a través de ella y a la vez promocionar a los que mejor han conseguido las metas propuestas. Los términos que han aparecido con mayor frecuencia son: valorar, sancionar, observar, seguir el proceso de construcción de conocimiento del alumno.

\section{BIBLIOGRAFÍA}

ANGUERA, M.T. y otros (1995). Métodos de investigación en psicopedagogía. Madrid, Síntesis.

ARIES, P. (1987). El Niño y la Vida Familiar en el Antiguo Régimen. Madrid, Taurus.

ASCH, S.E. (1955). “Opinions and social pressure". Scientific American, 193, 5, 31-35.

BANDURA, A. (1982). "Sel-efficacy mechanism in human agency". American Psychologist, 37, 122-147.

BANDURA, A. (1986). Social Foundations of Thought and Action. Englewood Cliffs, NJ, Prentice Hall.

BANDURA, A. (Ed.)(1995). Sel-efficacy in Changing Societies. New York, Cambridge University.

BARLETT, F.C. (1932). Remembering: An Study in Experimental and Social Psychology. Cambridge, Cambridge University Press.

BRUNER, J. (1985). Narrative and Paradigmatic Modes of Thought. Learning and Teaching the Ways of Knowing. Chicago: University of Chicago Press.

BUENDÍA, L. (1997). Métodos de investigación en Psicopedagogia. Madrid, McGraw-Hill.

CARMONA, M. (1994). Las creencias epistemológicas de los futuros profesores. En F. Lara Ortega (Comp). Psicología Evolutiva y de la Educación: Actas del IV Congreso de INFAD. Burgos, Universidad de Burgos.

CARMONA, M. (1995). Ideas de los profesores sobre el ayudar a pensar en el aula. II Congreso Internacional de Psicología y Educación. Madrid.

COSMIDES, L. (1989). "The logic of social change: Has natural selection shaped how humans reason? Studies with the Wason selection task". Cognition, 36, 187-196.

FENSTERMACHER, G.D. (1979). “A philosophical consideration of recent research on teacher effectiveness". En L.S. Shulman (Ed.) Review of Research in Education. 6, 157-185.

FENSTERMACHER, G.D. (1994). "The Knower and the known: The nature of Knowledge in research of teaching". Review of Research in Education. 20, 3-56.

GOODNOW, J., KNIGHT, R. y CASHMORE, J. (1988). Adult social cognition: Implications of parent's ideas for approaches to development. En $\mathbf{M}$. Perlmutter (Ed.). Minnesota symposia on child development. 18, 287-324. Hillsdale, NJ: Erlbaum.

HEIDER, F. (1958). The Psychology of Interpersonal Relations. Nueva York: Wiley.

HUMPHREY, N. (1987). La Reconquista de la Conciencia. Desarrollo de la Mente Humana. Méjico, F.C.E.

JODELET, D. (1983). Reflexions sur le traitement de la notion de representation sociale en psychlogie sociale. Actes de la tabble ronde internationale sur les representations. Communication-Information, número especial de octubre de 1983. 
JODELET, D. (1989). Civils et bredins: Representations sociales de la maladie nentale et rapport a la folieen milieu rural. Tesis de doctorado de estado. Paris.

KAGAN, D.M. (1990). "Ways of evaluating teacher cognition: Inferences concerning the goldilocks principle". Review of Educational Research. 60, 3, 419-469.

KAGAN, J. (1979). "Family experience and the child's development". American Psychologist, 34, 10, 886-891.

KAGAN, J. (1981). The second year. The emergence of self-awareness. Harvard University Press.

KESSEN, W. (1979). "The american child and other cultural inventions". American Psychologist, 34, 10, 815820.

LEWIN, K. (1935). A Dynamic Theory of Personality. Nueva York, McGrawHill.

MILLER, J.A. (1995). "Parents, attributions for their children". Child Development, 66, 1557-1584.

MOSCOVICI, S. (1983). The phenomenon of social representations. In R.M. Farr and S. Moscovici. (Eds.). Social Representations. Cambridge, University Press.

NESPOR, J. (1987). "The role of beliefs in the practice teaching". Journal of Curriculum Studies. 19. 317-328.

PAJARES, M.F. (1992). "Teacher's beliefs and educational research: cleaning up a messy construct". Review of Educational Research. 62, 3, 307-332.

PALACIOS, J. y MORENO, M.C. (1995). Sigel and Kim: In S. Harkness and C.M. Super (Eds.) Parents, cultural belief Systems. New York, Guilford Press (1995)

PALACIOS, J. y OLIVA, A. (1991). Ideas y Actitudes de Madres y Educadores sobre la Educación Infantil. (En prensa).

RIVIERE, A. (1991).Objetos con Mente. Madrid, Alianza.

SHERIFF, M. (1936). The Psychology of Social Norms. Nueva York, Harper.

SIGEL, I.E. (1986). Reflections on the belief-behavior connection: Lessons learned from a research program on parental belief systems and teaching strategies. In R.D. Ashmore and D.M. Brodzinsky (Eds.). Thinking About the Family: Views of Parents and Children. 35-36. Hillsdale, NJ: Lawrence, Erlbaum

VOLLMER, G. (1984) Mesocosmos and objective knowledge. in Concepts and Approaches to Evolutionary Epistemology: Towards an Evolutonary Theory of Knowledge. Ed. F. M. Wuketits, 69-121. Dordrecht, D. Reidel.

VYGOTSKY, L.S. (1978). El Desarrollo de los Procesos Psicológicos superiores. Barcelona, Crítica

WEINER, B. (1985). "An attributional theory of achievement motivation and emotion". Psychological Review. 92, 548-573.

WEINER, B. (1986). An attributional theory of motivation and emotion. New York, Springer Verlag.

WERTSCH, J. and Youniss, J. (1987). "Contextualizing the investigator: The case of developmental psychology". Human Development. 30, 18-31.

YARROW, I.J. (1979). Historical perpectives and futures directions in infant development. En J.D. Osofsky (Ed.). Handbook of infant development. New York: John Wiley and Sons.

YOUNG, K.T. (1990). "American conception of infant development from 1955 to 1984: What the experts are telling parents". Child Development, 61, 17-28. 


\section{RESUMEN}

Con el presente trabajo pretendemos hacer una conceptualización del término concepción. Tradicionalmente, las concepciones han sido consideradas por los investigadores como un constructo que engloba el conocimiento personal que los seres humanos poseen. Este término no es exclusivo, en el campo educativo el término concepción coexiste con términos tales como representación, creencias, ideas, teorias personales, actitudes, perspectivas...

Las concepciones de los sujetos se gestan sobre la base de la interacción de tres elementos: la naturaleza bio-antropológica, es decir, el prcoeso evolutivo seguido como especie y su influencia en el conocimiento; los aspectos históricos que permiten conservar y transmitir nuestras experiencias pasadas; el conjunto de elementos derivados de nuestra particularidad psicológica.

En la segunda parte del trabajo realizamos un estudio empírico para detectar cuáles son las concepciones de los profesores respecto a una serie de tópicos: qué evaluar, cuándo evaluar, cómo debe evaluarse, cómo mejorar la evaluación, qué es evaluar.. Para esto, se realizaron entrevistas semiestructuradas a profesores de secundaria. Las respuestas dadas se sometieron a análisis utilizando como soporte informático el programa Aquad (versión 3.0).

Palabras clave: Concepción, creencias, teoría personal, idea, evaluación, profesores de secundaria.

\section{ABSTRACT}

The present work tries to make a conceptualisation of the term conception. Traditionally, researchers have considered conceptions as a construct that includes the personal knowledge that tuman beings possess. It is not an exclusive term; in the educative field the term conception coexists with other designations as representation, beliefs, ideas, personal theories, attitudes, perspectives...

People's conceptions arise from the interaction of three elements: the bioantrhopological nature, that is, the evolutionary process followed as species and its influence in knowledge; historical aspects that allow to preserve and transmit our past experiences; the whole of elements derived from our psychological particularity.

In the second part of the work, we carried out an empirical study to detect which are the teachers conceptions in relation to the a series of topics: what 
to evaluate, when to evaluate, how we should evaluate, how to improve evaluation, what it is to evaluate. In order to accomplish this, we made halfstructured interviews on secondary teachers. The given answers underwent an analysis using the software Aquad (3.0 version).

Key words: Conceptions, beliefs, ideas, evaluation, secondary teachers, Aquad. 